\title{
Multiquartic functional equations
}

\author{
Abasalt Bodaghi ${ }^{1}$, Choonkil Park ${ }^{2 *}$ (D) and Oluwatosin T. Mewomo ${ }^{3}$
}

\author{
${ }^{*}$ Correspondence: \\ baak@hanyang.ac.kr \\ ${ }^{2}$ Research Institute for Natural \\ Sciences, Hanyang University, Seoul, \\ Korea \\ Full list of author information is \\ available at the end of the article
}

\begin{abstract}
In this paper, we study n-variable mappings that are quartic in each variable. We show that the conditions defining such mappings can be unified in a single functional equation. Furthermore, we apply an alternative fixed point method to prove the Hyers-Ulam stability for the multiquartic functional equations in the normed spaces. We also prove that under some mild conditions, every approximately multiquartic mapping is a multiquartic mapping.
\end{abstract}

MSC: 39B52; 39B72; 39B82; 46B03

Keywords: Banach space; Multiquartic mapping; Hyers-Ulam stability; Fixed point method

\section{Introduction}

A fundamental question in the theory of functional equations is as follows:

When is it true that a function that approximately satisfies a functional equation is close to an exact solution of the equation?

If this is the case, then we say that the equation is stable. The stability problem for the group homomorphisms was introduced by Ulam [1] in 1940. The first partial answer to Ulam's question in the case of Cauchy's equation or additive equation $A(x+y)=A(x)+A(y)$ in Banach spaces was given by Hyers [2] (stability involving a positive constant). Later the result of Hyers was significantly generalized by Aoki [3], T.M. Rassias [4] (stability incorporated with sum of powers of norms), Găvruța [5] (stability controlled by a general control function) and J.M. Rassias [6] (stability including mixed product-sum of powers of norms).

Let $V$ be a commutative group, let $W$ be a linear space, and let $n \geq 2$ be an integer. Recall from [7] that a mapping $f: V^{n} \longrightarrow W$ is called multiadditive if it is additive (i.e., it satisfies Cauchy's functional equation) in each variable. Furthermore, $f$ is said to be multiquadratic if it is quadratic (i.e., it satisfies quadratic the functional equation $Q(x+y)+Q(x-y)=$ $2 Q(x)+2 Q(y))$ in each variable [8]. Zhao et al. [9], showed that the system of functional equations defining a multiquadratic mapping can be unified in a single equation. Indeed, they proved that the mentioned mapping $f$ is multiquadratic if and only if the following relation holds:

$$
\sum_{s \in\{-1,1\}^{n}} f\left(x_{1}+s x_{2}\right)=2^{n} \sum_{j_{1}, j_{2}, \ldots, j_{n} \in\{1,2\}} f\left(x_{1 j_{1}}, x_{2 j_{2}}, \ldots, x_{n j_{n}}\right)
$$

(c) The Author(s) 2019. This article is distributed under the terms of the Creative Commons Attribution 4.0 International License (http://creativecommons.org/licenses/by/4.0/), which permits unrestricted use, distribution, and reproduction in any medium, provided you give appropriate credit to the original author(s) and the source, provide a link to the Creative Commons license, and indicate if changes were made. 
where $x_{j}=\left(x_{1 j}, x_{2 j}, \ldots, x_{n j}\right) \in V^{n}$ and $j \in\{1,2\}$. Ciepliński $[7,8]$ studied the Hyers-Ulam stability of multiadditive and multiquadratic mappings in Banach spaces (see also [9]). For more remarks on the Hyers-Ulam stability of some systems of functional equations, we refer to [10].

A mapping $f: V^{n} \longrightarrow W$ is called multicubic if it is cubic (i.e., it satisfies the cubic functional equation $C(2 x+y)+C(2 x-y)=2 C(x+y)+2 C(x-y)+12 C(x))$ in each variable [11]). In [12], the first author and Shojaee studied the Hyers-Ulam stability for multicubic mappings on normed spaces and also proved that a multicubic functional equation can be hyperstable, that is, every approximately multicubic mapping is multicubic. For other forms of cubic functional equations and their stabilities, we refer to [13-18].

The quartic functional equation

$$
\mathcal{Q}(x+2 y)+\mathcal{Q}(x-2 y)=4 \mathcal{Q}(x+y)+4 \mathcal{Q}(x-y)-6 \mathcal{Q}(x)+24 \mathcal{Q}(y)
$$

was introduced for the first time by Rassias [19]. It is easy to see that the function $\mathcal{Q}(x)=$ $a x^{4}$ satisfies (1.2). Thus, every solution of the quartic functional equation (1.2) is said to be a quartic function. The functional equation (1.2) was generalized by the first author and Kang in [20] and [21], respectively.

Motivated by definitions of multiadditive, multiqudratic, and multicubic mappings, we define multiquartic mappings and provide their characterization. In fact, we prove that every multiquartic mapping can be characterized by a single functional equation and vice versa. In addition, we investigate the Hyers-Ulam stability for multiquartic functional equations by applying the fixed point method, which was used for the first time by Baker in [22]. For more applications of this approach to the stability of multiadditive-quadratic mappings and multi-Cauchy-Jensen mappings in non-Archimedean spaces and Banach spaces, see [23-25].

\section{Characterization of multiquartic mappings}

Throughout this paper, $\mathbb{N}$ stands for the set of all positive integers, $\mathbb{N}_{0}:=\mathbb{N} \cup\{0\}, \mathbb{R}_{+}:=$ $[0, \infty), n \in \mathbb{N}$. For any $l \in \mathbb{N}_{0}, m \in \mathbb{N}, t=\left(t_{1}, \ldots, t_{m}\right) \in\{-2,2\}^{m}$, and $x=\left(x_{1}, \ldots, x_{m}\right) \in V^{m}$, we write $l x:=\left(l x_{1}, \ldots, l x_{m}\right)$ and $t x:=\left(t_{1} x_{1}, \ldots, t_{m} x_{m}\right)$, where $r a$ stands, as usual, for the $r$ th power of an element $a$ of the commutative group $V$.

Let $n \in \mathbb{N}$ with $n \geq 2$, and let $x_{i}^{n}=\left(x_{i 1}, x_{i 2}, \ldots, x_{i n}\right) \in V^{n}, i \in\{1,2\}$. We denote $x_{i}^{n}$ by $x_{i}$ when there is no risk of ambiguity. For $x_{1}, x_{2} \in V^{n}$ and $p_{i} \in \mathbb{N}_{0}$ with $0 \leq p_{i} \leq n$, put $\mathcal{N}=$ $\left\{\left(N_{1}, N_{2}, \ldots, N_{n}\right) \mid N_{j} \in\left\{x_{1 j} \pm x_{2 j}, x_{1 j}, x_{2 j}\right\}\right\}$, where $j \in\{1, \ldots, n\}$ and $i \in\{1,2\}$. Consider the following subset of $\mathcal{N}$ :

$$
\mathcal{N}_{\left(p_{1}, p_{2}\right)}^{n}:=\left\{\mathfrak{N}_{n}=\left(N_{1}, N_{2}, \ldots, N_{n}\right) \in \mathcal{N} \mid \operatorname{Card}\left\{N_{j}: N_{j}=x_{i j}\right\}=p_{i}(i \in\{1,2\})\right\} .
$$

For $r \in \mathbb{R}$, we put $r \mathcal{N}_{\left(p_{1}, p_{2}\right)}^{n}=\left\{r \mathfrak{N}_{n}: \mathfrak{N}_{n} \in \mathcal{N}_{\left(p_{1}, p_{2}\right)}^{n}\right\}$. In this section, we assume that $V$ and $W$ are vector spaces over the rationals. We say a mapping $f: V^{n} \longrightarrow W$ is $n$-multiquartic or multiquartic if $f$ is quartic in each variable (see equation (1.2)). For such mappings, we use the following notations:

$$
\begin{aligned}
& f\left(\mathcal{N}_{\left(p_{1}, p_{2}\right)}^{n}\right):=\sum_{\mathfrak{N}_{n} \in \mathcal{N}_{\left(p_{1}, p_{2}\right)}^{n}} f\left(\mathfrak{N}_{n}\right), \\
& f\left(\mathcal{N}_{\left(p_{1}, p_{2}\right)}^{n}, z\right):=\sum_{\mathfrak{N}_{n} \in \mathcal{N}_{\left(p_{1}, p_{2}\right)}^{n}} f\left(\mathfrak{N}_{n}, z\right) \quad(z \in V) .
\end{aligned}
$$


For all $x_{1}, x_{2} \in V^{n}$, we consider the equation

$$
\sum_{t \in\{-2,2\}^{n}} f\left(x_{1}+t x_{2}\right)=\sum_{p_{2}=0}^{n} \sum_{p_{1}=0}^{n-p_{2}} 4^{n-p_{1}-p_{2}}(-6)^{p_{1}} 24^{p_{2}} f\left(\mathcal{N}_{\left(p_{1}, p_{2}\right)}^{n}\right) .
$$

By a mathematical computation we can check that the mapping $f: \mathbb{R}^{n} \longrightarrow \mathbb{R}$ defined as $f\left(z_{1}, \ldots, z_{n}\right)=\prod_{j=1}^{n} a_{j} z_{j}^{4}$ satisfies (2.2). Thus this equation is said to be the multiquartic functional equation.

We denote $\left(\begin{array}{l}n \\ k\end{array}\right)=n ! /(k !(n-k) !)$ (the binomial coefficients) for all $n, k \in \mathbb{N}$ with $n \geq k$.

Let $0 \leq k \leq n-1$. Put $\mathcal{K}_{k}=\left\{{ }_{k} x:=\left(0, \ldots, 0, x_{j_{1}}, 0, \ldots, 0, x_{j_{k}}, 0, \ldots, 0\right) \in V^{n}\right\}$, where $1 \leq j_{1}<$ $\cdots<j_{k} \leq n$. In other words, $\mathcal{K}_{k}$ is the set of all vectors in $V^{n}$ whose exactly $k$ components are nonzero.

We will show that a mapping $f: V^{n} \longrightarrow W$ satisfies the functional equation (2.2) if and only if it is multiquartic. For this, we need the following lemma.

Lemma 2.1 If a mapping $f: V^{n} \longrightarrow W$ satisfies equation (2.2), then $f(x)=0$ for any $x \in V^{n}$ with at least one component equal to zero.

Proof We argue by induction on $k$ that for each ${ }_{k} x \in \mathcal{K}_{k}, f\left({ }_{k} x\right)=0$ for $0 \leq k \leq n-1$. For $k=0$, by putting $x_{1}=x_{2}=(0, \ldots, 0)$ in $(2.2)$ we have

$$
\begin{aligned}
& 2^{n} f(0, \ldots, 0) \\
& \quad=\sum_{p_{2}=0}^{n} \sum_{p_{1}=0}^{n-p_{2}} 4^{n-p_{1}-p_{2}}(-6)^{p_{1}} 24^{p_{2}}\left(\begin{array}{c}
n \\
n-p_{1}-p_{2}
\end{array}\right)\left(\begin{array}{c}
p_{1}+p_{2} \\
p_{1}
\end{array}\right) 2^{n-p_{1}-p_{2}} f(0, \ldots, 0) .
\end{aligned}
$$

It is easily verified that

$$
\left(\begin{array}{c}
n-k \\
n-k-p_{1}-p_{2}
\end{array}\right)\left(\begin{array}{c}
p_{1}+p_{2} \\
p_{1}
\end{array}\right)=\left(\begin{array}{c}
n-k \\
p_{2}
\end{array}\right)\left(\begin{array}{c}
n-k-p_{2} \\
p_{1}
\end{array}\right)
$$

for $0 \leq k \leq n-1$. Using (2.4) for $k=0$, we compute the right-hand side of (2.3) as follows:

$$
\begin{aligned}
& \sum_{p_{2}=0}^{n} \sum_{p_{1}=0}^{n-p_{2}} 4^{n-p_{1}-p_{2}}(-6)^{p_{1}} 24^{p_{2}}\left(\begin{array}{c}
n \\
n-p_{1}-p_{2}
\end{array}\right)\left(\begin{array}{c}
p_{1}+p_{2} \\
p_{1}
\end{array}\right) 2^{n-p_{1}-p_{2}} f(0, \ldots, 0) \\
& \quad=2^{n}\left[\sum_{p_{2}=0}^{n}\left(\begin{array}{c}
n \\
p_{2}
\end{array}\right) 12^{p_{2}} \sum_{p_{1}=0}^{n-p_{2}}\left(\begin{array}{c}
n-p_{2} \\
p_{1}
\end{array}\right) 4^{n-p_{1}-p_{2}}(-3)^{p_{1}}\right] f(0, \ldots, 0) \\
& =2^{n}\left[\sum_{p_{2}=0}^{n}\left(\begin{array}{c}
n \\
p_{2}
\end{array}\right) 12^{p_{2}}(4-3)^{n-p_{2}}\right] f(0, \ldots, 0) \\
& =2^{n}(12+1)^{n} f(0, \ldots, 0)=26^{n} f(0, \ldots, 0) .
\end{aligned}
$$

From relations (2.3) an (2.5) it follows that $f(0, \ldots, 0)=0$. Assume that for each ${ }_{k-1} x \in \mathcal{K}_{k-1}$, $f\left({ }_{k-1} x\right)=0$. We show that if ${ }_{k} x \in \mathcal{K}_{k}$, then $f\left({ }_{k} x\right)=0$. By a suitable replacement in (2.2) we 
get

$$
\begin{aligned}
2^{n} f\left({ }_{k} x\right) & =\sum_{p_{2}=0}^{n-k} \sum_{p_{1}=0}^{n-k-p_{2}} 4^{n-p_{1}-p_{2}}(-6)^{p_{1}} 24^{p_{2}}\left(\begin{array}{c}
n-k \\
n-k-p_{1}-p_{2}
\end{array}\right)\left(\begin{array}{c}
p_{1}+p_{2} \\
p_{1}
\end{array}\right) 2^{n-p_{1}-p_{2}} f\left({ }_{k} x\right) \\
& =2^{n} 4^{k}\left[\sum_{p_{2}=0}^{n-k}\left(\begin{array}{c}
n-k \\
p_{2}
\end{array}\right) 12^{p_{2}} \sum_{p_{1}=0}^{n-k-p_{2}}\left(\begin{array}{c}
n-k-p_{2} \\
p_{1}
\end{array}\right) 4^{\left.n-k-p_{1}-p_{2}(-3)^{p_{1}}\right] f\left({ }_{k} x\right)}\right. \\
& =2^{n} 4^{k}\left[\sum_{p_{2}=0}^{n-k}\left(\begin{array}{c}
n-k \\
p_{2}
\end{array}\right) 12^{p_{2}}(4-3)^{n-k-p_{2}}\right] f\left({ }_{k} x\right) \\
& =2^{n} 4^{k}(12+1)^{n-k} f\left({ }_{k} x\right)=2^{n+2 k} 13^{n-k} f\left({ }_{k} x\right) .
\end{aligned}
$$

Hence $f\left({ }_{k} x\right)=0$. This shows that $f(x)=0$ for any $x \in V^{n}$ with at least one component equal to zero.

We now prove the main result of this section.

Theorem 2.2 A mapping $f: V^{n} \longrightarrow W$ is multiquartic if and only if it satisfies the functional equation (2.2).

Proof Let $f$ be multiquartic. We prove that $f$ satisfies the functional equation (2.2) by induction on $n$. For $n=1$, it is trivial that $f$ satisfies the functional equation (1.2). If (2.2) is valid for some positive integer $n>1$, then

$$
\begin{aligned}
& \sum_{t \in\{-2,2\}^{n+1}} f\left(x_{1}^{n+1}+t x_{2}^{n+1}\right) \\
= & 4 \sum_{t \in\{-2,2\}^{n}} f\left(x_{1}^{n}+t x_{2}^{n}, x_{1 n+1}+x_{2 n+1}\right)+4 \sum_{t \in\{-2,2\}^{n}} f\left(x_{1}^{n}+t x_{2}^{n}, x_{1 n+1}-x_{2 n+1}\right) \\
& -6 \sum_{t \in\{-2,2\}^{n}} f\left(x_{1}^{n}+t x_{2}^{n}, x_{1 n+1}\right)+24 \sum_{t \in\{-2,2\}^{n}} f\left(x_{1}^{n}+t x_{2}^{n}, x_{2 n+1}\right) \\
= & 4 \sum_{p_{2}=0}^{n} \sum_{p_{1}=0}^{n-p_{2}} \sum_{t \in\{-2,2\}} 4^{n-p_{1}-p_{2}}(-6)^{p_{1}} 24^{p_{2}} f\left(\mathcal{N}_{\left(p_{1}, p_{2}\right)}^{n}, x_{1 n+1}+t x_{2 n+1}\right) \\
& -6 \sum_{p_{2}=0}^{n} \sum_{p_{1}=0}^{n-p_{2}} 4^{n-p_{1}-p_{2}}(-6)^{p_{1}} 24^{p_{2}} f\left(\mathcal{N}_{\left(p_{1}, p_{2}\right)}^{n}, x_{1 n+1}\right) \\
& +24 \sum_{p_{2}=0}^{n} \sum_{p_{1}=0}^{n-p_{2}} 4^{n-p_{1}-p_{2}}(-6)^{p_{1}} 24^{p_{2}} f\left(\mathcal{N}_{\left(p_{1}, p_{2}\right)}^{n}, x_{2 n+1}\right) \\
= & \sum_{p_{2}=0}^{n+1} \sum_{p_{1}=0}^{n+1-p_{2}} 4^{n+1-p_{1}-p_{2}}(-6)^{p_{1}} 24^{p_{2}} f\left(\mathcal{N}_{\left(p_{1}, p_{2}\right)}^{n+1}\right) .
\end{aligned}
$$

This means that (2.2) holds for $n+1$.

Conversely, suppose that $f$ satisfies the functional equation (2.2). Fix $j \in\{1, \ldots, n\}$. Set

$$
\begin{aligned}
f^{*}\left(x_{1 j}, x_{2 j}\right):= & f\left(x_{11}, \ldots, x_{1 j-1}, x_{1 j}+x_{2 j}, x_{1 j+1}, \ldots, x_{1 n}\right) \\
& +f\left(x_{11}, \ldots, x_{1 j-1}, x_{1 j}-x_{2 j}, x_{1 j+1}, \ldots, x_{1 n}\right)
\end{aligned}
$$


and

$$
f^{*}\left(x_{2 j}\right):=f\left(x_{11}, \ldots, x_{1 j-1}, x_{2 j}, x_{1 j+1}, \ldots, x_{1 n}\right) .
$$

Putting $x_{2 k}=0$ for all $k \in\{1, \ldots, n\} \backslash\{j\}$ in (2.2) and using Lemma 2.1, we get

$$
\begin{aligned}
2^{n-1}[ & f\left(x_{11}, \ldots, x_{1 j-1}, x_{1 j}+2 x_{2 j}, x_{1 j+1}, \ldots, x_{1 n}\right) \\
& \left.+f\left(x_{11}, \ldots, x_{1 j-1}, x_{1 j}-2 x_{2 j}, x_{1 j+1}, \ldots, x_{1 n}\right)\right] \\
= & \sum_{p_{1}=0}^{n-1}\left(\begin{array}{c}
n-1 \\
p_{1}
\end{array}\right) 4^{n-p_{1}}(-6)^{p_{1}} 2^{n-p_{1}-1} f^{*}\left(x_{1 j}, x_{2 j}\right) \\
& +\sum_{p_{1}=1}^{n}\left(\begin{array}{c}
n-1 \\
p_{1}-1
\end{array}\right) 4^{n-p_{1}}(-6)^{p_{1}} 2^{n-p_{1}} f\left(x_{11}, \ldots, x_{1 n}\right) \\
& +\sum_{p_{1}=1}^{n}\left(\begin{array}{c}
n-1 \\
p_{1}-1
\end{array}\right) 4^{n-p_{1}}(-6)^{p_{1}-1} 2^{n-p_{1}} f^{*}\left(x_{2 j}\right) \\
= & 42^{n-1} \sum_{p_{1}=0}^{n-1}\left(\begin{array}{c}
n-1 \\
p_{1}
\end{array}\right) 4^{n-1-p_{1}}(-3)^{p_{1}} f^{*}\left(x_{1 j}, x_{2 j}\right) \\
& \quad 6 \times 2^{n-1} \sum_{p_{1}=0}^{n-1}\left(\begin{array}{c}
n-1 \\
p_{1}
\end{array}\right) 4^{n-1-p_{1}}(-3)^{p_{1}} f\left(x_{11}, \ldots, x_{1 n}\right) \\
& +24 \times 2^{n-1} \sum_{p_{1}=0}^{n-1}\left(\begin{array}{c}
n-1 \\
p_{1}
\end{array}\right) 4^{n-1-p_{1}}(-3)^{p_{1}} f^{*}\left(x_{2 j}\right) \\
& 4 \times 2^{n-1} f^{*}\left(x_{1 j}, x_{2 j}\right)-6 \times 2^{n-1} f\left(x_{11}, \ldots, x_{1 n}\right)+24 \times 2^{n-1} f^{*}\left(x_{2 j}\right) .
\end{aligned}
$$

Note that we have used the fact that $\sum_{p_{1}=0}^{n-1}\left(\begin{array}{c}n-1 \\ p_{1}\end{array}\right) 4^{n-1-p_{1}}(-3)^{p_{1}}=(4-3)^{n-1}=1$ in the above computations. So this relation implies that $f$ is quartic in the $j$ th variable. Since $j$ is arbitrary, we obtain the desired result.

\section{Stability results for the functional equation (2.2)}

For two sets $X$ and $Y$, we denote by $Y^{X}$ the set of all mappings from $X$ to $Y$, In this section, we wish to prove the Hyers-Ulam stability of the functional equation (2.2) in normed spaces. The proof is based on a fixed point result that can be derived from [26, Theorem 1]. To state it, we introduce three hypotheses:

(A1) $Y$ is a Banach space, $\mathcal{S}$ is a nonempty set, $j \in \mathbb{N}, g_{1}, \ldots, g_{j}: \mathcal{S} \longrightarrow \mathcal{S}$, and $L_{1}, \ldots, L_{j}: \mathcal{S} \rightarrow \mathbb{R}_{+}$

(A2) $\mathcal{T}: Y^{\mathcal{S}} \longrightarrow Y^{\mathcal{S}}$ is an operator satisfying the inequality

$$
\|\mathcal{T} \lambda(x)-\mathcal{T} \mu(x)\| \leq \sum_{i=1}^{j} L_{i}(x)\left\|\lambda\left(g_{i}(x)\right)-\mu\left(g_{i}(x)\right)\right\|, \quad \lambda, \mu \in Y^{\mathcal{S}}, x \in \mathcal{S},
$$

(A3) $\Lambda: \mathbb{R}_{+}^{\mathcal{S}} \longrightarrow \mathbb{R}_{+}^{\mathcal{S}}$ is an operator defined as

$$
\Lambda \delta(x):=\sum_{i=1}^{j} L_{i}(x) \delta\left(g_{i}(x)\right) \quad \delta \in \mathbb{R}_{+}^{\mathcal{S}}, x \in \mathcal{S} .
$$


Here we highlight the following theorem. which is a fundamental result in fixed point theory [26, Theorem 1]. This result plays a key tool to obtain our objective in this paper.

Theorem 3.1 Let (A1)-(A3) hold and suppose that a function $\theta: \mathcal{S} \longrightarrow \mathbb{R}_{+}$and a mapping $\phi: \mathcal{S} \longrightarrow Y$ fulfill the following two conditions:

$$
\|\mathcal{T} \phi(x)-\phi(x)\| \leq \theta(x), \quad \theta^{*}(x):=\sum_{l=0}^{\infty} \Lambda^{l} \theta(x)<\infty \quad(x \in \mathcal{S})
$$

Then there exists a unique fixed point $\psi$ of $\mathcal{T}$ such that

$$
\|\phi(x)-\psi(x)\| \leq \theta^{*}(x) \quad(x \in \mathcal{S}) .
$$

Moreover, $\psi(x)=\lim _{l \rightarrow \infty} \mathcal{T}^{l} \phi(x)$ for all $x \in \mathcal{S}$.

For a given the mapping $f: V^{n} \longrightarrow W$, we define the difference operator $\Gamma f: V^{n} \times$ $V^{n} \longrightarrow W$ by

$$
\Gamma f\left(x_{1}, x_{2}\right):=\sum_{t \in\{-2,2\}^{n}} f\left(x_{1}+t x_{2}\right)-\sum_{p_{2}=0}^{n} \sum_{p_{1}=0}^{n-p_{2}} 4^{n-p_{1}-p_{2}}(-6)^{p_{1}} 24^{p_{2}} f\left(\mathcal{N}_{\left(p_{1}, p_{2}\right)}^{n}\right)
$$

for all $x_{1}, x_{2} \in V^{n}$, where $f\left(\mathcal{N}_{\left(p_{1}, p_{2}\right)}^{n}\right)$ is defined in (2.1).

Definition 3.2 Let $V$ be a vector space, let $W$ be a normed space, and let $\varphi: V^{n} \times$ $V^{n} \longrightarrow \mathbb{R}_{+}$be a function. We call that a mapping $f: V^{n} \longrightarrow W$ is approximately $\varphi\left(x_{1}, x_{2}\right)$ multiquartic or briefly approximately $\varphi$-multiquartic if

$$
\left\|\Gamma f\left(x_{1}, x_{2}\right)\right\| \leq \varphi\left(x_{1}, x_{2}\right) \quad\left(x_{1}, x_{2} \in V^{n}\right) .
$$

In addition, the mapping $f: V^{n} \longrightarrow W$ is called even in the $j$ th variable if

$$
f\left(z_{1}, \ldots, z_{j-1},-z_{j}, z_{j+1}, \ldots, z_{n}\right)=f\left(z_{1}, \ldots, z_{j-1}, z_{j}, z_{j+1}, \ldots, z_{n}\right), \quad z_{1}, \ldots, z_{n} \in V
$$

We say that a mapping $f: V^{n} \longrightarrow W$ satisfies the approximately $\varphi$-even-zero conditions if

(i) $f$ is approximately $\varphi$-multiquartic;

(ii) $f$ is even in each variable;

(iii) $f(x)=0$ for any $x \in V^{n}$ with at least one component equal to 0 .

Remark 3.3 We note that the approximately $\varphi$-even-zero conditions for the mapping $f$ : $V^{n} \longrightarrow W$ do not imply that $f$ is multiquartic. Indeed, there are plenty of examples of $f$ with the mentioned conditions that are not multiquartic. Here we give a concrete example for $n=2$. Let $(\mathcal{A},\|\cdot\|)$ be a Banach algebra. Fix a unit vector $a_{0}$ in $\mathcal{A}$. Define the mapping $h: \mathcal{A} \times \mathcal{A} \longrightarrow \mathcal{A}$ by $h(x, y)=\|x\|\|y\| a_{0}$ for $x, y \in \mathcal{A}$. Clearly, $h$ satisfies conditions (ii) and (iii). Define $\varphi: \mathcal{A}^{2} \times \mathcal{A}^{2} \longrightarrow \mathbb{R}_{+}$by

$$
\varphi\left(\left(a_{1}, b_{1}\right),\left(a_{2}, b_{2}\right)\right)=c\left(\left\|a_{1}\right\|+\left\|a_{2}\right\|\right)\left(\left\|b_{1}\right\|+\left\|b_{2}\right\|\right), \quad\left(a_{1}, b_{1}\right),\left(a_{2}, b_{2}\right) \in \mathcal{A}^{2},
$$


where $c \geq 1172$. A computation shows that

$$
\left\|\Gamma h\left(\left(a_{1}, b_{1}\right),\left(a_{2}, b_{2}\right)\right)\right\| \leq \varphi\left(\left(a_{1}, b_{1}\right),\left(a_{2}, b_{2}\right)\right) .
$$

Hence $h$ satisfies the approximately $\varphi$-even-zero conditions, but it is not a 2-multiquartic mapping.

In the next theorem, we prove the Hyers-Ulam stability of the functional equation (2.2).

Theorem 3.4 Let $s \in\{-1,1\}$, let $V$ be a linear space, and let $W$ be a Banach space. Suppose that $f: V^{n} \longrightarrow W$ satisfies approximately $\varphi$-even-zero conditions and

$$
\lim _{l \rightarrow \infty}\left(\frac{1}{2^{4 n s}}\right)^{l} \varphi\left(2^{s l} x_{1}, 2^{s l} x_{2}\right)=0
$$

for all $x_{1}, x_{2} \in V^{n}$. If

$$
\Phi(x)=\frac{1}{2^{n+2 n(s+1)}} \sum_{l=0}^{\infty}\left(\frac{1}{2^{4 n s}}\right)^{l} \varphi\left(0,2^{s l+\frac{s-1}{2}} x\right)<\infty
$$

for all $x \in V^{n}$, then there exists a unique multiquartic mapping $\mathfrak{Q}: V^{n} \longrightarrow W$ such that

$$
\|f(x)-\mathfrak{Q}(x)\| \leq \Phi(x)
$$

for all $x \in V^{n}$.

Proof Replacing $\left(x_{1}, x_{2}\right)$ by $(0, x)$ in $(3.1)$ and using the assumptions, we have

$$
\left\|2^{n} f(2 x)-\sum_{p_{2}=0}^{n}\left(\begin{array}{c}
n \\
p_{2}
\end{array}\right) 4^{n-p_{2}} 24^{p_{2}} 2^{n-p_{2}} f(x)\right\| \leq \varphi(0, x)
$$

for all $x \in V^{n}$. We note that $\sum_{p_{2}=0}^{n}\left(\begin{array}{c}n \\ p_{2}\end{array}\right) 4^{n-p_{2}} 24^{p_{2}} 2^{n-p_{2}}=(8+24)^{n}=32^{n}$. Inequality (3.5) implies that

$$
\left\|f(2 x)-2^{4 n} f(x)\right\| \leq \frac{1}{2^{n}} \varphi(0, x) \quad\left(x \in V^{n}\right) .
$$

For each $x \in V^{n}$, set

$$
\xi(x):=\frac{1}{2^{n+2 n(s+1)}} \varphi\left(0,2^{\frac{s-1}{2}} x\right), \quad \text { and } \quad \mathcal{T} \xi(x):=\frac{1}{2^{4 n s}} \xi\left(2^{s} x\right) \quad\left(\xi \in W^{V^{n}}\right) .
$$

Then relation (3.6) can be written as

$$
\|f(x)-\mathcal{T} f(x)\| \leq \xi(x) \quad\left(x \in V^{n}\right) .
$$

Define $\Lambda \eta(x):=\frac{1}{2^{4 n s}} \eta\left(2^{s} x\right)$ for $\eta \in \mathbb{R}_{+}^{V^{n}}$ and $x \in V^{n}$. We now see that $\Lambda$ has the form described in (A3) with $\mathcal{S}=V^{n}, g_{1}(x)=2^{s} x$, and $L_{1}(x)=\frac{1}{2^{4 n s}}$ for $x \in V^{n}$. Furthermore, for all 
$\lambda, \mu \in W^{V^{n}}$ and $x \in V^{n}$, we get

$$
\|\mathcal{T} \lambda(x)-\mathcal{T} \mu(x)\|=\left\|\frac{1}{2^{4 n s}}\left[\lambda\left(2^{s} x\right)-\mu\left(2^{s} x\right)\right]\right\| \leq L_{1}(x)\left\|\lambda\left(g_{1}(x)\right)-\mu\left(g_{1}(x)\right)\right\| .
$$

This relation shows that hypothesis (A2) holds. By induction on $l$ we can check for any $l \in \mathbb{N}_{0}$ and $x \in V^{n}$ that

$$
\Lambda^{l} \xi(x):=\left(\frac{1}{2^{4 n s}}\right)^{l} \xi\left(2^{s l} x\right)=\frac{1}{2^{n+2 n(s+1)}}\left(\frac{1}{2^{4 n s}}\right)^{l} \varphi\left(0,2^{s l+\frac{s-1}{2}} x\right)
$$

for all $x \in V^{n}$. Relations (3.3) and (3.8) ensure that all assumptions of Theorem 3.1 are satisfied. Hence there exists a unique mapping $\mathfrak{Q}: V^{n} \longrightarrow W$ such that

$$
\mathfrak{Q}(x)=\lim _{l \rightarrow \infty}\left(\mathcal{T}^{l} f\right)(x)=\frac{1}{2^{4 n s}} \mathfrak{Q}\left(2^{s} x\right) \quad\left(x \in V^{n}\right)
$$

and (3.4) holds. We will show that

$$
\left\|\Gamma\left(\mathcal{T}^{l} f\right)\left(x_{1}, x_{2}\right)\right\| \leq\left(\frac{1}{2^{4 n s}}\right)^{l} \varphi\left(2^{s l} x_{1}, 2^{s l} x_{2}\right)
$$

for all $x_{1}, x_{2} \in V^{n}$ and $l \in \mathbb{N}_{0}$. We argue by induction on $l$. Inequality (3.9) is valid for $l=0$ by (3.1). Assume that (3.9) is true for $l \in \mathbb{N}_{0}$. Then

$$
\begin{aligned}
\| & \Gamma\left(\mathcal{T}^{l+1} f\right)\left(x_{1}, x_{2}\right) \| \\
& =\left\|\sum_{t \in\{-2,2\}^{n}}\left(\mathcal{T}^{l+1} f\right)\left(x_{1}+t x_{2}\right)-\sum_{p_{2}=0}^{n} \sum_{p_{1}=0}^{n-p_{2}} 4^{n-p_{1}-p_{2}}(-6)^{p_{1}} 24^{p_{2}}\left(\mathcal{T}^{l+1} f\right)\left(\mathcal{N}_{\left(p_{1}, p_{2}\right)}^{n}\right)\right\| \\
& =\frac{1}{2^{4 n s}}\left\|\sum_{t \in\{-2,2\}^{n}}\left(\mathcal{T}^{l} f\right)\left(2^{s}\left(x_{1}+t x_{2}\right)\right)-\sum_{p_{2}=0}^{n} \sum_{p_{1}=0}^{n-p_{2}} 4^{n-p_{1}-p_{2}}(-6)^{p_{1}} 24^{p_{2}}\left(\mathcal{T}^{l} f\right)\left(2^{s} \mathcal{N}_{\left(p_{1}, p_{2}\right)}^{n}\right)\right\| \\
& =\frac{1}{2^{4 n s}}\left\|\Gamma\left(\mathcal{T}^{l} f\right)\left(2^{s} x_{1}, 2^{s} x_{2}\right)\right\| \leq\left(\frac{1}{2^{4 n s}}\right)^{l+1} \varphi\left(2^{s(l+1)} x_{1}, 2^{s(l+1)} x_{2}\right)
\end{aligned}
$$

for all $x_{1}, x_{2} \in V^{n}$. Letting $l \rightarrow \infty$ in (3.9) and applying (3.2), we obtain that $\Gamma \mathfrak{Q}\left(x_{1}, x_{2}\right)=0$ for all $x_{1}, x_{2} \in V^{n}$. So the mapping $\mathfrak{Q}$ satisfies (2.2) and thus is multiquartic. This finishes the proof.

Let $A$ be a nonempty set, let $(X, d)$ bea metric space, let $\psi \in \mathbb{R}_{+}^{A^{n}}$, and let $\mathcal{F}_{1}, \mathcal{F}_{2}$ be operators mapping a nonempty set $D \subset X^{A}$ into $X^{A^{n}}$. We say that the operator equation

$$
\mathcal{F}_{1} \varphi\left(a_{1}, \ldots, a_{n}\right)=\mathcal{F}_{2} \varphi\left(a_{1}, \ldots, a_{n}\right)
$$

is $\psi$-hyperstable if every $\varphi_{0} \in D$ satisfying the inequality

$$
d\left(\mathcal{F}_{1} \varphi_{0}\left(a_{1}, \ldots, a_{n}\right), \mathcal{F}_{2} \varphi_{0}\left(a_{1}, \ldots, a_{n}\right)\right) \leq \psi\left(a_{1}, \ldots, a_{n}\right), \quad a_{1}, \ldots, a_{n} \in A
$$

fulfils (3.10); this definition is introduced in [27]. Under some conditions, the functional equation (2.2) can be hyperstable as the following corollary shows. 
Corollary 3.5 Let $\delta>0$. Suppose that $\chi_{k j}>0$ for $k \in\{1,2\}$ and $j \in\{1, \ldots, n\}$ fulfill $\sum_{k=1}^{2} \sum_{j=1}^{n} \chi_{k j} \neq 4 n$. Let $V$ be a normed space, and let $W$ be a Banach space. Iff $: V^{n} \longrightarrow W$ satisfies approximately $\prod_{k=1}^{2} \prod_{j=1}^{n}\left\|x_{k j}\right\|^{\chi_{k j}} \delta$-even-zero conditions, then it is multiquartic.

In the following corollaries, which are direct consequences of Theorem 3.4, we show that the functional equation (2.2) is stable. Since the proofs are routine, we include them without proofs.

Corollary 3.6 Let $\lambda \in \mathbb{R}$ with $\lambda \neq 4 n$. Let $V$ be a normed space, and let $W$ be a Banach space.Iff $: V^{n} \longrightarrow W$ satisfies approximately $\sum_{k=1}^{2} \sum_{j=1}^{n}\left\|x_{k j}\right\|^{\lambda}$-even-zero conditions, then there exists a unique multiquartic mapping $\mathfrak{Q}: V^{n} \longrightarrow W$ such that

$$
\|f(x)-\mathfrak{Q}(x)\| \leq \begin{cases}\frac{2^{4 n}}{2^{5 n}\left(2^{4 n}-2^{\lambda}\right)} \sum_{j=1}^{n}\left\|x_{1 j}\right\|^{\lambda}, & \lambda<4 n, \\ \frac{1}{2^{n}\left(2^{\lambda}-2^{4 n}\right)} \sum_{j=1}^{n}\left\|x_{1 j}\right\|^{\lambda}, & \lambda>4 n,\end{cases}
$$

for all $x=x_{1} \in V^{n}$.

Corollary 3.7 Let $\delta>0$. Let $V$ be a normed space, and let $W$ be a Banach space. If $f: V^{n} \longrightarrow W$ satisfies approximately $\delta$-even-zero conditions, then there exists a unique multiquartic mapping $\mathfrak{Q}: V^{n} \longrightarrow W$ such that

$$
\|f(x)-\mathfrak{Q}(x)\| \leq \frac{2^{4 n}}{2^{5 n}\left(2^{4 n}-1\right)} \delta
$$

for all $x \in V^{n}$.

\section{Conclusions}

We have applied an alternative fixed point method to prove the Hyers-Ulam stability for the multiquartic functional equations in the normed spaces, and we have proved that under some mild conditions, every approximately multiquartic mapping is a multiquartic mapping.

\section{Acknowledgements}

The authors sincerely thank the anonymous reviewer for her/his careful reading, constructive comments, and fruitful suggestions that substantially improved the manuscript.

\section{Funding}

Not applicable.

Availability of data and materials

Not applicable.

Competing interests

The authors declare that they have no competing interests.

Authors' contributions

All authors conceived of the study, participated in its design and coordination, drafted the manuscript, participated in the sequence alignment, and read and approved the final manuscript.

\section{Author details}

'Department of Mathematics, Garmsar Branch, Islamic Azad University, Garmsar, Iran. ${ }^{2}$ Research Institute for Natural Sciences, Hanyang University, Seoul, Korea. ${ }^{3}$ School of Mathematics, Statistics and Computer Science, University of KwaZulu-Natal, Durban, South Africa. 


\section{Publisher's Note}

Springer Nature remains neutral with regard to jurisdictional claims in published maps and institutional affiliations.

Received: 26 March 2019 Accepted: 24 July 2019 Published online: 02 August 2019

\section{References}

1. Ulam, S.M.: Problems in Modern Mathematics. Science Editions. Wiley, New York (1964)

2. Hyers, D.H.: On the stability of the linear functional equation. Proc. Natl. Acad. Sci. USA 27, 222-224 (1941)

3. Aoki, T.: On the stability of the linear transformation in Banach spaces. J. Math. Soc. Jpn. 2, $64-66$ (1950)

4. Rassias, T.M.: On the stability of the linear mapping in Banach spaces. Proc. Am. Math. Soc. 72, 297-300 (1978)

5. Găvruţa, P:: A generalization of the Hyers-Ulam-Rassias stability of approximately additive mappings. J. Math. Anal. Appl. 184, 431-436 (1994)

6. Rassias, J.M.: On approximately of approximately linear mappings by linear mappings. J. Funct. Anal. 46, 126-130 (1982)

7. Ciepliński, K.: Generalized stability of multi-additive mappings. Appl. Math. Lett. 23, 1291-1294 (2010)

8. Ciepliński, K.: On the generalized Hyers-Ulam stability of multi-quadratic mappings. Comput. Math. Appl. 62, $3418-3426(2011)$

9. Zhao, X., Yang, X., Pang, C.T.: Solution and stability of the multiquadratic functional equation. Abstr. Appl. Anal. 2013, Article ID 415053 (2013)

10. Brzdęk, J., Ciepliński, K.: Remarks on the Hyers-Ulam stability of some systems of functional equations. Appl. Math Comput. 219, 4096-4105 (2012)

11. Jun, K., Kim, H.: The generalized Hyers-Ulam-Rassias stability of a cubic functional equation. J. Math. Anal. Appl. 274, 267-278 (2002)

12. Bodaghi, A., Shojaee, B.: On an equation characterizing multi-cubic mappings and its stability and hyperstability. arXiv:1907.09378v1

13. Bodaghi, A.: Intuitionistic fuzzy stability of the generalized forms of cubic and quartic functional equations. J. Intell. Fuzzy Syst. 30, 2309-2317 (2016)

14. Bodaghi, A., Moosavi, S.M., Rahimi, H.: The generalized cubic functional equation and the stability of cubic Jordan *-derivations. Ann. Univ. Ferrara 59, 235-250 (2013)

15. Eghbali, N., Rassias, J.M., Taheri, M.: On the stability of a $k$-cubic functional equation in intuitionistic fuzzy $n$-normed spaces. Results Math. 70, 233-248 (2016)

16. Eskandani, Z., Rassias, J.M.: Stability of general A-cubic functional equations in modular spaces. Rev. R. Acad. Cienc. Exactas Fís. Nat., Ser. A Mat. 112, 425-435 (2018). https://doi.org/10.1007/s13398-017-0388-5

17. Jun, K., Kim, H.: On the Hyers-Ulam-Rassias stability of a general cubic functional equation. Math. Inequal. Appl. 6 289-302 (2003)

18. Rassias, J.M.: Solution of the Ulam stability problem for cubic mappings. Glas. Mat. Ser. III 36, 63-72 (2001)

19. Rassias, J.M.: Solution of the Ulam stability problem for quartic mappings. Glas. Mat. Ser. III 34, 243-252 (1999)

20. Bodaghi, A.: Stability of a quartic functional equation. Sci. World J. 2014, Article ID 752146 (2014). https://doi.org/10.1155/2014/752146

21. Kang, D:: On the stability of generalized quartic mappings in quasi- $\beta$-normed spaces. J. Inequal. Appl. 2010, Article ID 198098 (2010). https://doi.org/10.1155/2010/198098

22. Baker, J.A.: The stability of certain functional equations. Proc. Am. Math. Soc. 112, 729-732 (1991)

23. Bahyrycz, A., Ciepliński, K., Olko, J.: On an equation characterizing multi-additive-quadratic mappings and its Hyers-Ulam stability. Appl. Math. Comput. 265, 448-455 (2015)

24. Bahyrycz, A., Ciepliński, K., Olko, J.: On an equation characterizing multi-Cauchy-Jensen mappings and its Hyers-Ulam stability. Acta Math. Sci. Ser. B Engl. Ed. 35, 1349-1358 (2015)

25. Bahyrycz, A., Ciepliński, K., Olko, J.: On Hyers-Ulam stability of two functional equations in non-Archimedean spaces. J. Fixed Point Theory Appl. 18, 433-444 (2016)

26. Brzdęk, J., Chudziak, J., Páles, Z:: A fixed point approach to stability of functional equations. Nonlinear Anal. 74 6728-6732 (2011)

27. Brzdęk, J., Ciepliński, K.: Hyperstability and superstability. Abstr. Appl. Anal. 2013, Article ID 401756 (2013)

\section{Submit your manuscript to a SpringerOpen ${ }^{\circ}$ journal and benefit from:}

- Convenient online submission

- Rigorous peer review

- Open access: articles freely available online

- High visibility within the field

- Retaining the copyright to your article

Submit your next manuscript at $\downarrow$ springeropen.com 